\title{
Packing to leave
}

An essential feature of the life cycle of hepatitis $\mathrm{B}$ virus (HBV) is the packaging of the viral RNA pre-genome (pgRNA) into virions. It has been hypothesized that pgRNA is specifically packaged into viral nucleocapsids; however, the HBV assembly pathway remains incompletely understood. In a recent study, Patel, White et al. discover that the HBV pgRNA contains specific nucleotide motifs that mediate interactions with the viral capsid protein to drive nucleocapsid assembly.

First, the authors used systematic evolution of ligands by exponential enrichment (SELEX) against the purified HBV capsid protein to identify RNA sequences that bind

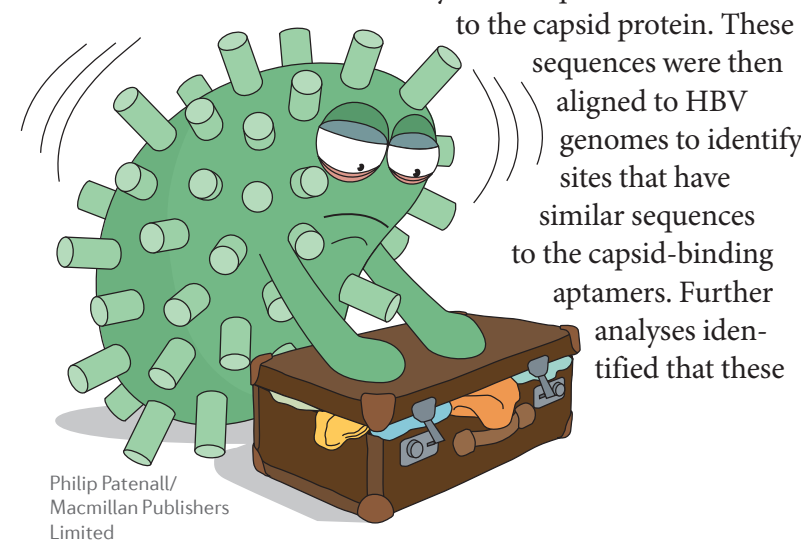

sites are predicted to form stem-loop structures and have a purine-rich motif (RGAG; in which $\mathrm{R}$ represents G or A).

Next, the authors designed oligoribonucleotides that contained the predicted stem-loop structures and saw that these oligoribonucleotides bind to the HBV capsid protein and trigger the assembly of virus-like particles (VLPs). Using cryo-electron microscopy, an asymmetric RNA feature was identified in these VLPs below the capsid protein shell, which the authors claim represents a complex between the identified packaging signals and the viral capsid shell.

These results suggest a new model of HBV assembly.

This study provides new evidence that specific protein-RNA interactions are important for the assembly of HBV virions.

\section{Ashley York}

ORIGINAL ARTICLE Patel, N., White, S. J. et al. HBV RNA pre-genome encodes specific motifs that mediate interactions with the viral core protein that promote nucleocapsid assembly. Nat. Microbiol. 2, 17098 (2017) 